\title{
L'expression des gènes d'antigène variable chez les trypanosomes africains
}

\section{Étienne Pays}

\section{Remerciements}

Je remercie le professeur M. Steinert pour ses commentaires sur ce manuscrit. Les recherches du laboratoire sont financées par le Fonds de la recherche scientifique médicale, et des contrats avec la CEE (TDS-M-23B), Solvay et $\mathrm{C}^{\text {ie }}$ (Bruxelles) et l'OMS (UNDP/World Bank/WHO Special Programme for Research and Training in Tropical Diseases).

\section{ADRESSE}

E. Pays : chef de travaux. Département de biologie moléculaire, université libre de Bruxelles, 67, rue des Chevaux, B 1640

Les trypanosomes africains sont responsables de graves infections chroniques chez l'homme et le bétail. Une des stratégies employées par ces parasites pour échapper aux défenses immunitaires de leur hôte consiste à changer continuellement de revêtement antigénique de surface. L'analyse des mécanismes génétiques assurant cette variation révèle des particularités étonnantes. Le génome du trypanosome abrite une collection de plusieurs centaines de gènes $d$ 'antigène variable différents, dont beaucoup sont localisés dans de petits chromosomes qui ne semblent servir qu'à cet effet. Un seul de ces gènes est exprimé à tout moment dans un des quelques 5 à 20 télomères compétents. Dans le sang du mammifère, le changement de manteau antigénique est dû à deux types de mécanismes : l'activation alternative des différents sites d'expression potentiels, ou des recombinaisons (principalement la conversion génique) entre le gène $d$ 'antigène actif et $d$ 'autres gènes du répertoire. L'utilisation successive de ces différents mécanismes assure une évolution rapide des répertoires de gènes, ainsi qu'une certaine programmation dans l'ordre d'expression des antigènes.

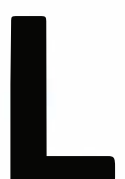

es trypanosomes africains, tels que Trypanosoma brucei, sont véhiculés par des glossines ("mouche tsé-tsé ") qui les inoculent à divers mammifères lors de repas sanguins. Les trypanosomes présents dans les glandes salivaires de la mouche (forme "métacyclique ") sont préparés à ce nouvel environnement, et prolifèrent aussitôt dans le sang (forme "sanguicole"). Cette prolifération peut se poursuivre pendant de très longues périodes, en dépit de la réponse immunitaire de l'hôte, ce qui permet l'infestation d'autres glossines et le bouclage du cycle. Dans l'intestin moyen de l'insecte, le trypano- some se différencie en forme " procyclique ", et se multiplie jusqu'à la maturation finale en forme métacyclique. Les différentes formes du parasite se caractérisent notamment par leur recouvrement de surface. Ainsi que le résume le schéma de la figure 1, les trypanosomes métacycliques et sanguicoles expriment une protéine de surface appelée VSG (pour variant surface glycoprotein), qui est remplacée par la procycline chez les trypanosomes procycliques. Ces deux protéines ont une structure très différente, mais toutes deux sont ancrées à la membrane plasmique par un radical glycophospholipidique, et toutes deux forment un manteau 
de surface homogène et dense (pour une revue récente, voir [1]). Alors que la fonction de la procycline n'est pas connue, il est clair qu'au moins un des rôles du VSG est d'assurer la défense du parasite contre le système immunitaire de l'hôte. En effet, cette molécule possède la double propriété d'être à la fois très immunogène et très variable, ce qui déroute continuellement la réponse immune. C'est à l'étude des mécanismes génétiques présidant à l'expression de cet antigène qu'est consacré cet article (pour plus de détails, voir [2], [3]).

\section{Les gènes de VSG : structure et localisation}

Comme cela est schématisé dans la figure 2, les gènes de VSG se distinguent par la présence d'une région extrêmement variable codant pour les deux tiers $\mathrm{N}$-terminaux de la protéine, en amont d'une région conservée correspondant à un domaine $\mathrm{C}$ terminal plus structuré. C'est grâce à des sondes d'ADN correspondant à ce domaine conservé (bloc d'homologie 3') que l'on a pu estimer le nombre total de gènes. Cette estimation oscille autour de mille copies. Il faut toutefois se garder d'utiliser ces données pour évaluer la taille du répertoire d'antigènes, car elles ne tiennent pas compte de l'existence de gènes non fonctionnels ou de gènes dupliqués ; d'autre part, les mécanismes de réarrangements de séquences dont il sera question plus loin assu-

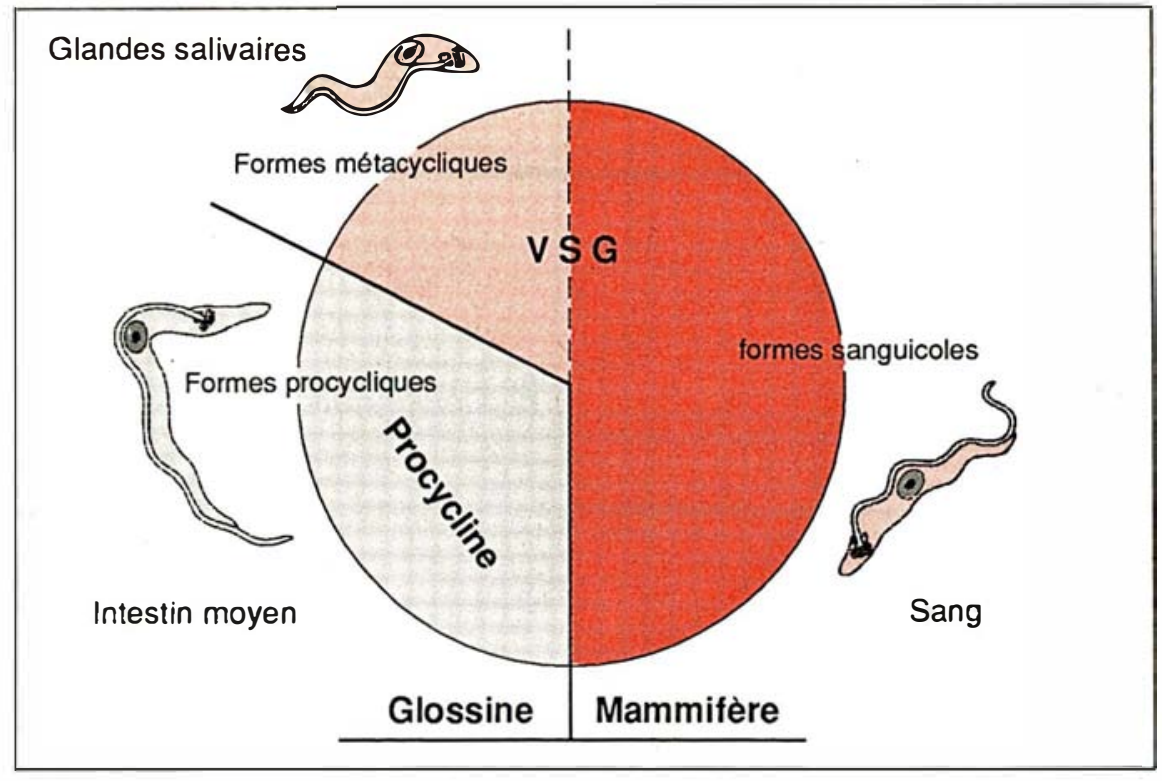

Figure 1. Protéines majeures de surface au cours du cycle parasitaire de Trypanosoma brucei.

rent un potentiel quasi illimité d'expansion du répertoire.

En fonction de leur environnement génomique, les gènes de VSG peuvent être répartis en deux catégories, les gènes télomériques et les gènes non télomériques (figure $3 A$ et $B$, page suivante).

Beaucoup sont localisés en bout de chromosome, et sont donc télomériques. Généralement, c'est l'extrémité 3' des gènes qui pointe vers le télomère. Les gènes télomériques sont souvent uniques et peu conservés entre souches différentes de trypano- somes. Ils sont flanqués de deux régions étendues d'ADN simple*, dépourvues de sites de restriction (régions barren***). La région barren 3' se compose de motifs typiquement télomériques, en particulier l'hexanucléotide TTAGGG qui est aussi retrouvé à l'extrémité des chromosomes humains. La région barren 5 ' est

\footnotetext{
* ADN simple : $A D N$ de séquence monotone, constituée de la répétition un grand nombre de fois de très courts motifs identiques.

** Barren: aride, stérile.
}

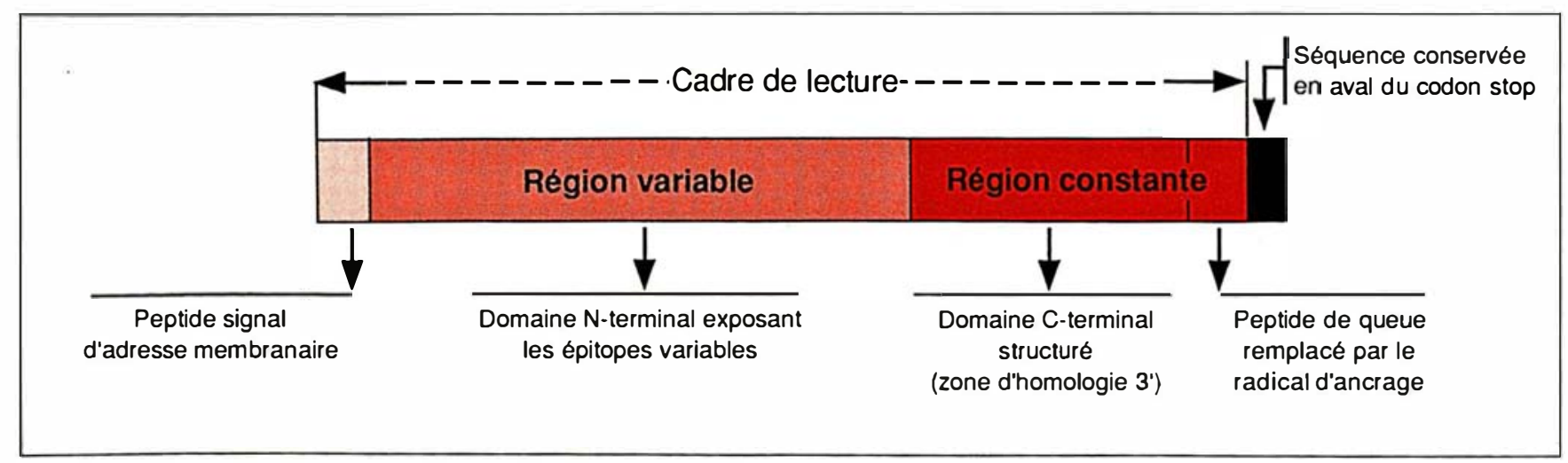

Figure 2. Structure générale des gènes de VSG. 


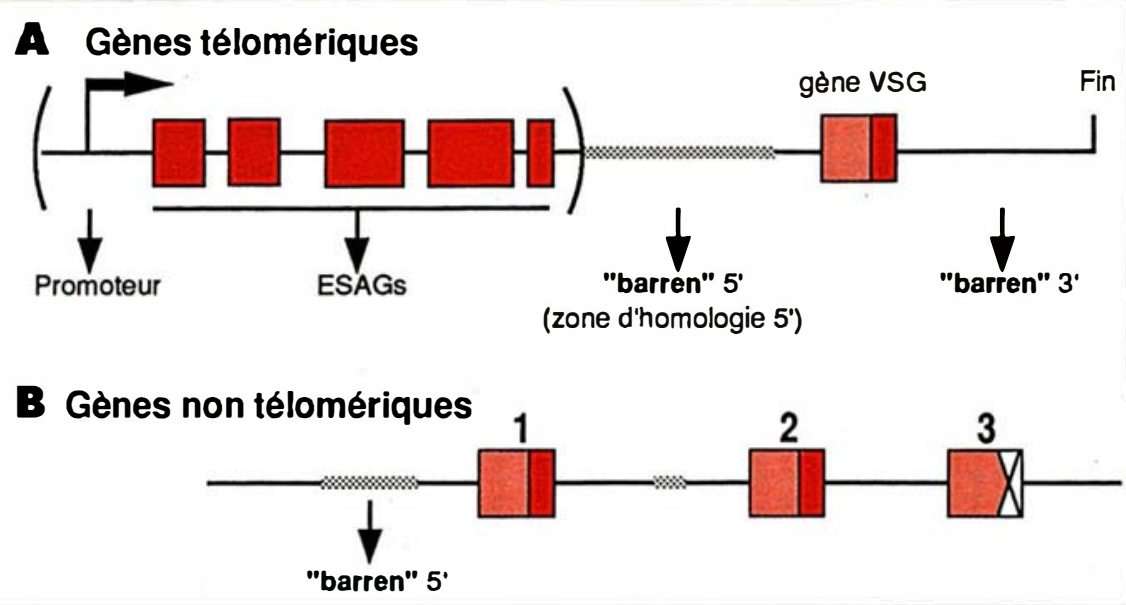

1. Braun-Breton C, Pereira da Siva LH. Modulation de l'activité biologique des protéines de surface chez les protozoaires hémoparasites. médecine/sciences 1989 ; 5 : 736-43.

2. Borst P. Discontinuous transcription and antigenic variation in trypanosomes. Annu Rev Biochem 1986 ; 55 : 701-32.

3. Pays E, Steinert M. Control of antigen gene expression in African trypanosomes. Annu Rev Genet 1988 : 22 : 107-26.

4. Pays E, Tebabi P, Pays A, et al. The genes and transcripts of an antigen gene expression site from $T$. brucei. Cell 1989 ; 57 : 835-45.

5. Pays E, Coquelet H, Tebabi $\mathrm{P}$, et al. $T$. brucei : constitutive activity of the VSG and procyclin gene promoters. EMBO J 1990 ; $9: 1035-40$.

6. Greaves DR, Borst P. T. brucei variantspecific glycoprotein genc chromatin is sensitive to single-strand-spccific endonuclcase digestion. J Mol Biol 1987 ; 197 : 471-83.

7. Gibson WC, Swinkels BW, Borst P. Post-transcriptional control of the differential expression of phosphoglycerate kinase genes in T. brucei. J Mol Biol 1988; 201 : 315-25.

8. Ehlers B, Czichos J, Overath P. RNA turnover in T. brucei. Mol Cell Biol 1987 ; $7: 1242-9$

9. Pays E, Coquclet H, Pays A, Tebabi P, Steinert M. T. brucei : post-transcriptional control of the variable surface glycoprotein gene expression site. Mol Cell Biol 1989 ; 9 : 4018-21.

10. Pays E. DNA recombinations and transposition in trypanosomes. In : Kingsman AJ, Kingsman SM, Chater KF, eds Transposition. Cambridge : Cambridge University Press, 1988 : 301-42.

11. Van der Werf A, Van Assel S, Aerts D, Steinert M, Pays E. Telomere interactions may condition the programming of antigen expression in $T$. brucei. EMBO J 1990 ; sous presse.

12. Pays E. Pseudogenes, chimaeric genes and the timing of antigen variation in Afrcan trypanosomes. Trends in Genet 1989 ; 5 : 389-91.

Figure 3. Organisation chromosomique des gènes de VSG. Le schéma en A symbolise l'environnement d'un gène télomérique. Ce gène est localisé à 5-10 kilobases de la fin d'un chromosome, et est flanqué de deux régions de DNA simple et répété (régions barren). La région en amont de la "barren " $5^{\prime}$ peut, ou non (parenthèses), contenir d'autres gènes (ESAGs, pour " expression site-associated genes "), ainsi qu'un promoteur de transcription. Si ces séquences sont présentes, l'ensemble du télomère, d'une longueur de 50 à 70 kilobases, constitue un site d'expression potentiel pour gène de VSG. En $B$ est représenté un modèle symbolique d'alignement de gènes non télomériques. Le gène 1 est flanqué des zones d'homologie requises pour se recombiner avec le site d'expression (zigzags pour les répétitions $5^{\prime}$, boîte noire pour la zone conservée $3^{\prime}$ ), le gène 2 possède des blocs d'homologie insuffisants, et le gène 3 est doublement non fonctionnel, car il ne possède pas d'homologie $5^{\prime}$ et son cadre de lecture est interrompu par des codons stops. Aussi bien en $A$ qu'en $B$, les éléments figurés ne sont pas à l'échelle.

constituée par la répétition, sur plusieurs kilobases, d'un motif d'environ 70 nucléotides particulièrement " recombinogène ". Cette région sert de bloc d'homologie 5' dans les mécanismes recombinatoires décrits plus loin. En amont de cette région, deux situations contrastées peuvent se présenter. Si le gène est localisé à l'extrémité de l'un des nombreux minichromosomes d'environ 50 à 150 kilobases qui caractérisent $T$. bru$c e i$, il semble que la région barren 5' se prolonge en amont sans rencontrer d'autres types de séquences. En effet, mis à part certains éléments transposables, aucun autre gène que ceux de VSG n'a pu être localisé dans les minichromosomes. Il semblerait donc que ces derniers ne servent que de réservoirs à gènes de VSG. L'autre situation est celle d'un gène de VSG localisé dans un des sites d'expression potentiels. Dans ce cas, ainsi que l'indique la figure $3 \mathrm{~A}$ (région entre parenthèses), d'autres gènes sont pré- sents immédiatement en amont de la région barren 5'. Nous allons y revenir dans la prochaine section.

Si de nombreux gènes de VSG sont télomériques, la plupart sont groupés plus à l'intérieur des chromosomes. La figure $3 B$ illustre différents exemples de gènes non télomériques. Dans le cas du gène 1 , les blocs d'homologie 5' et 3' sont tous deux présents. Ces blocs sont constitués des mêmes éléments que dans le cas des gènes télomériques, mais la région barren 5' est toujours moins étendue. Le nombre de motifs de 70 nucléotides varie d'un gène à l'autre : dans nos exemples, il est très réduit (gène 2) ou absent (gène 3). De même, la zone d'homologie 3' peut être amputée (gène 2) ou être non fonctionnelle par suite de mutations (gène 3 ). Il est fréquent que les gènes non télomériques fassent partie de familles de séquences apparentées, dont certains membres peuvent coder pour des antigènes différents ou être non fonc- 
tionnels. C'est parmi les gènes de VSG non télomériques que se retrouvent ceux qui sont les plus conservés entre souches de trypanosomes.

\section{Expression des gènes de VSG}

En dépit de l'étendue du répertoire, normalement, un seul gène de VSG est exprimé à tout moment. Ceci semble destiné à assurer la formation d'un manteau antigénique homogène : dans les cas exceptionnels où deux antigènes sont synthétisés en même temps, le trypanosome choisit rapidement l'un des deux. Il se pourrait que des associations hétérogènes de VSG ne protègent pas efficacement le parasite contre l'action lytique du sérum, même non immun.

Le gène de VSG est transcrit dans un site d'expression télomérique. Les raisons de cette localisation particulière sont totalement inconnues. Différents sites d'expression possibles coexistent dans un même clone cellulaire ; leur nombre n'est pas connu avec précision, mais se situe vraisemblablement entre 5 et 20. L'ARN polymérase qui transcrit le gène de VSG se caractérise par une résistance inhabituelle à l' $\alpha$-amanitine. Très curieusement, cette particularité est partagée par les gènes de procycline, en dépit de différences importantes dans la structure et l'activité des deux types d'unités de transcription. Tout aussi curieusement, la transcription du gène de VSG est inversement corrélée à la manifestation d'une activité qui protège partiellement certains sites de restriction télomériques contre la digestion par les endonucléases spécifiques. Cette activité, encore non définie, pourrait aboutir à une modification particulière et spécifique de l'ADN télomérique, qui ne s'opèrerait donc qu'en cas d'inactivité du télomère. Le promoteur de transcription se situe loin en amont du gène de VSG, et d'autres gènes, appelés ESAGs pour expression site-associated genes, font partie de la même unité de transcription [4] (figure 3A). La fonction de ces gènes, ainsi que la signification de leur expression conjointe avec le gène de VSG, ne peuvent, à l'heure actuelle, qu'être l'objet de conjectures. Le seul indice existant est une $\mathrm{m} / \mathrm{s} n^{\circ} 6$ vol. 6 , juin 90 homologie de séquence significative entre le gène ESAG 4 et les gènes connus d'adénylate cyclase eucaryotique, ce qui peut être mis en relation avec la découverte récente, chez $T$. brucci, d'une activité d'adénylate cyclase typiquement sanguicole. Cependant, la relation entre adénylate cyclase et VSG est loin d'être claire ; le seul lien établi à ce jour est une stimulation spécifique de l'enzyme par des concentrations de calcium qui provoquent aussi le détachement du VSG via l'hydrolyse du pont glycophospholipidique le reliant à la membrane. Par ailleurs, le gène ESAG 4, comme certains autres ESAGs, fait partie d'une famille multigénique dont d'autres membres sont exprimés à différents stades du cycle parasitaire, ce qui montre que des gènes similaires peuvent être transcrits sous la direction de régions régulatrices différentes. Il semble que la transcription en une unité comprenant plusieurs gènes ne soit pas un caractère propre au site d'expression de gènes de VSG, mais commun à la plupart des gènes connus des trypanosomes. Ce phénomène est peut-être à relier au mécanisme particulier d'épissage en trans des longs transcrits primaires [2]*.

\section{Contrôles de l'expression du gène de VSG}

La transcription du site d'expression subit au moins trois types de contrôle.

Dans le sang du mammifère, un mécanisme encore non compris assure la sélectivité de l'activité d'un seul site d'expression par cellule. Cependant, quoiqu'exceptionnellement, deux sites peuvent être actifs simultanément, ce qui démontre que plusieurs promoteurs de transcription de gènes de VSG coexistent dans la génome. Ceci est confirmé par la comparaison des séquences nucléotidiques de la région du promoteur, entre le télomère transcrit ou un télomère non transcrit du même clone : ces séquences sont pratiquement

\footnotetext{
* Épissage en trans : jonction d'exons provenant d'unités de transcription différentes. Un tel mécanisme intervient au niveau des transcrits de VSG qui sont épissés en trans à un "miniexon" commun codé par une séquence génomique sans lien avec les gènes VSG.
}

identiques et, de plus, peuvent toutes deux diriger l'expression de gènes bactériens lors d'essais d'expression transitoire [5]. Il est donc clair qu'au stade sanguicole un mécanisme contrôle et sélectionne l'activité d'un promoteur de transcription parmi plusieurs candidats potentiels. C'est probablement le même mécanisme qui intervient dans l'activation alternative de différents sites d'expression au cours de la prolifération du parasite. Les spéculations actuelles au sujet de ce mécanisme d'exclusion font intervenir de possibles interactions entre le télomère actif et la matrice nucléaire, ce que la modification de l'ADN télomérique, uniquement observée en cas d'inactivité du gène, pourrait empêcher [6].

Un autre type de contrôle de l'activité du site d'expression s'opère au cours du cycle parasitaire. Lorsque la forme sanguicole se différencie en forme procyclique, soit par passage dans la glossine, soit par culture à basse température in vitro, la transcription du gène de VSG s'arrête très rapidement. Cette inactivation va de pair avec la stimulation immédiate de la synthèse de l'ARN messager de procycline, et donc le remplacement du VSG par la procycline à la surface du parasite. De manière frappante, le contrôle de l'activité de ces deux unités de transcription s'avère similaire, tout en opérant de façon inverse : dans les deux cas, l'environnement adverse du parasite conduit à un blocage rapide de l'élongation de la transcription, couplé à l'accélération de la dégradation des transcrits. L'activité des promoteurs de transcription respectifs ne semble cependant pas affectée [5]. Ce type de contrôle, agissant essentiellement au niveau de la stabilité des transcrits, pourrait également assurer la différenciation d'autres fonctions cellulaires au cours du cycle parasitaire [7]. Les facteurs intervenant dans ce processus ne sont pas connus, mais pourraient dépendre d'une synthèse protéique [8].

Un dernier type de contrôle s'observe dans les glandes salivaires de la mouche. A ce stade, l'activité de sites d'expression de gènes de VSG reprend. Toutefois, ce phénomène se distingue totalement de ce que l'on observe dans la forme sanguicole. 
Tout d'abord, il ne concerne pas le site d'expression actif juste avant le passage du parasite dans la mouche. Ce dernier ne se réactive en effet qu'après l'injection du trypanosome dans le circuit sanguin du mammifère, sans doute par simple levée du bloquage de l'élongation de la transcription [9]. Ensuite, ce phénomène est manifestement induit par un facteur de la mouche, qui déclenche simultanément, chez tous les trypanosomes arrivés à ce stade du cycle, l'activation de l'un des 10-20 sites d'expression spécifiquement "métacycliques". Enfin, l'activité de ces sites s'éteint progressivement lorsque les trypanosomes sont injectés dans le sang, ce qui démontre clairement que cette activité ne dépend pas de facteurs présents dans le sang. Les sites où cette expression particulière se produit semblent propres aux télomères des plus grands chromosomes. Ils pourraient différer des sites sanguicoles par l'absence de la région barren 5' et des ESAGs. Cet isolement génétique par rapport aux sites d'expression sanguicoles pourrait être responsable de la conservation relative du répertoire antigénique métacyclique, qui se limite à un peu plus d'une dizaine de types différents. Toutefois, les types antigéniques métacycliques peuvent aussi être réexprimés plus tard, au cours de l'infection chronique dans le sang; cette réexpression passe très probablement par des mécanismes d'activation génique typiquement sanguicoles, que nous allons décrire dans la section suivante.

\section{Mécanismes de variation des gènes de VSG}

La variation antigénique des trypanosomes sanguicoles résulte du fonctionnement de deux mécanismes fondamentalement différents : le changement de site d'expression et le changement de gène de VSG au sein d'un même site d'expression. Ces mécanismes sont illustrés dans la figure 4.

Le premier, baptisé " activation in situ ", a déjà été évoqué plus haut. Il consiste en l'utilisation alternée de différents sites d'expression, sans réarrangement apparent de l'ADN, ni au niveau du gène de VSG, ni au niveau du promoteur de transcription. Étant donné que les différents sites d'expression potentiels portent des gènes de VSG différents, cette alternance entraîne un changement de type antigénique. Ce mécanisme s'observe principalement en début d'infection sanguicole, probablement parce qu'à ce stade aucune réponse immunitaire anti-VSG ne fait obstacle à la forte propension d'au moins plusieurs sites d'expression à s'activer spontanément.

Le second mécanisme regroupe différents types de recombinaison de l'ADN, qui permettent un remplacement, total ou partiel, du gène de VSG actif par un autre. Ce remplacement peut être un échange par

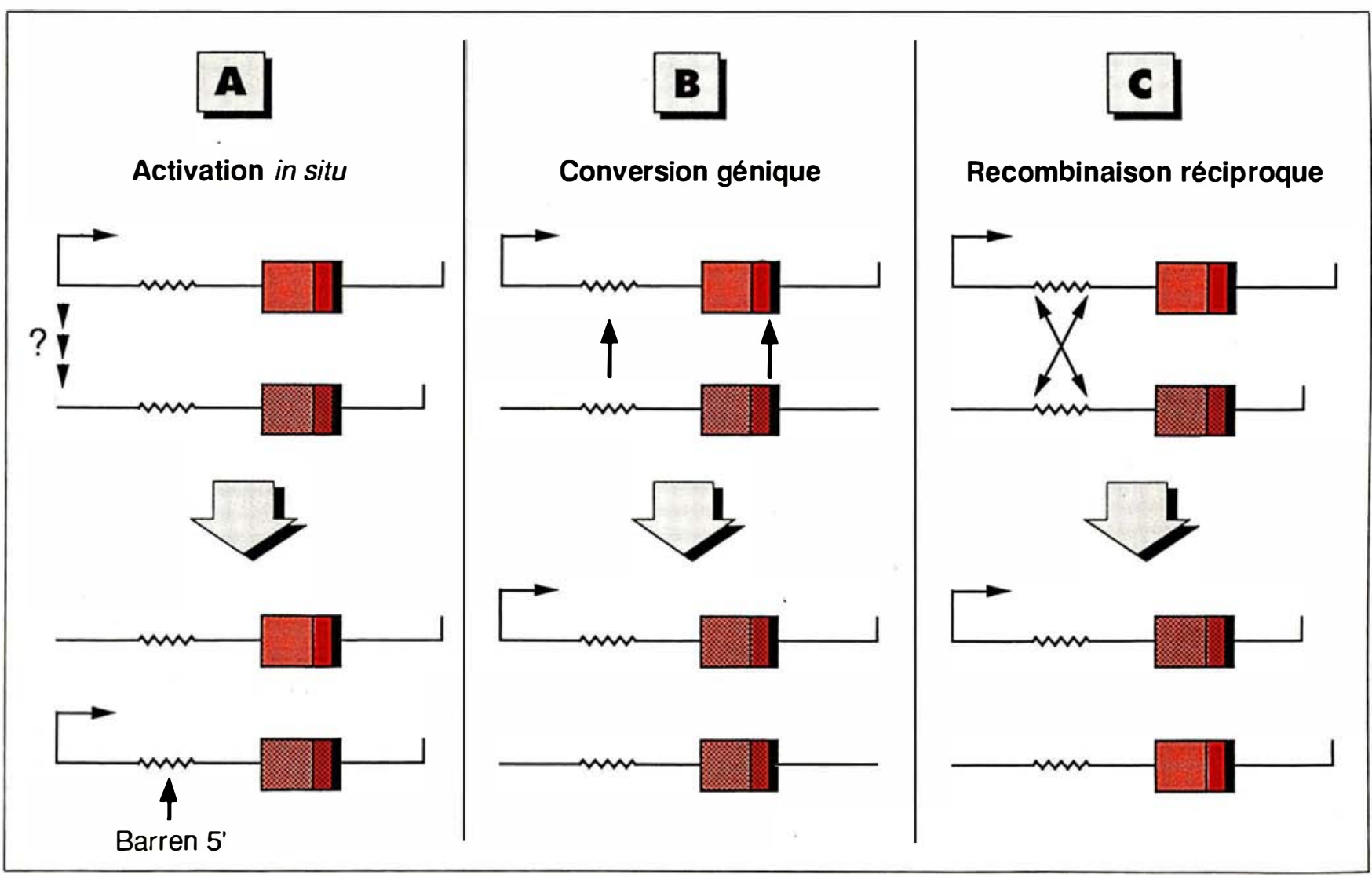

Figure 4. Mécanismes de variation antigénique. La flèche horizontale symbolise la transcription et définit le site 
recombinaison réciproque entre télomères, bien que ceci n'ait été observé que très rarement. Le plus souvent, le remplacement s'opère par conversion génique, c'est-à-dire par recombinaison non réciproque. Dans ce cas, le gène est remplacé par la copie d'un autre gène (voir [10] pour plus de détails). Ce mécanisme recombinatoire est très fréquent entre membres de familles multigéniques. Chez le trypanosome, il présente la caractéristique d'être particulièrement flexible : les étendues de conversion génique peuvent aller de quelques nucléotides à plusieurs dizaines de kilobases. Le plus fréquemment, cette étendue va du bloc d'homologie 5' a u bloc 3', c'est-à-dire des motifs répétés de 70 nucléotides, généralement situés environ 1,5 kilobase en amont du gène, jusqu'à la région conservée en fin du gène. Lorsque la conversion se termine à l'intérieur d'un gène, tel qu'on l'observe tard en cours d'infection (voir ci-dessous), l'événement recombinatoire conduit à la formation de gènes composites, ce qui peut étendre le répertoire de gènes. Selon des estimations récentes, la fréquence des conversions géniques entre gènes de VSG serait particulièrement élevée. Ceci pourrait être attribué à la présence des régions barren : la région 5' est un lieu privilégié de recombinaison, probablement par sa répétition et par la séquence particulière des motifs, et la région 3', c'est-à-dire le télomère proprement dit, semble, comme dans les autres types cellulaires, être le siège d'interactions entre chromosomes, ce qui favorise aussi les recombinaisons [11].

\section{Programmation de la variation antigénique}

Le répertoire de types antigéniques ne s'exprime pas totalement au hasard. Certains antigènes sont typiquement précoces, tandis que d'autres ne s'observent que rarement, ou exceptionnellement. Il est clair que cette programmation permet au parasite de prolonger l'infection, en évitant un épuisement trop rapide du leurre antigénique. Ce phénomène ne semble pas dépendre de la nature des antigènes ou de leurs gènes, mais plutôt de l'environne$\mathrm{m} / \mathrm{s} n^{\circ} 6$ vol. 6 , juin 90 ment génomique de ces derniers [12]. Lorsqu'ils sont situés dans des sites d'expression potentiels, les gènes de VSG ont une forte probabilité d'expression, et il n'est donc pas étonnant que les gènes s'exprimant précocement soient télomériques. De même, les gènes télomériques non situés dans des sites d'expression, comme le sont ceux présents dans les minichromosomes, apparaissent facilement activables par recombinaison avec le site d'expression, probablement grâce au niveau élevé d'homologie partagé entre télomères. Ensuite peuvent s'exprimer les gènes non télomériques, qui ne peuvent avoir accès au site d'expression qu'en tant que matrices pour la synthèse d'une copie, c'est-à-dire en tant que donneurs dans des événements de conversion génique. Il existe toute une gamme de possibilités d'expression en ce qui concerne ces gènes. S'ils sont flanqués des deux blocs d'homologie nécessaires pour la recombinaison avec le site d'expression (gène 1 dans la figure $3 B$ ), une conversion complète est possible d'un bloc à l'autre. Dans ce cas, il semble que la probabilité d'expression du gène dépende de l'ampleur des homologies. Parfois, le bloc d'homologie 5' peut être insuffisant (gène 2) ou même absent (gène 3), et le bloc d'homologie 3' peut être tronqué ou interrompu par des codons stops. Dans ces différents cas, le gène ne peut être exprimé facilement, soit parce que les homologies sont insuffisantes pour permettre la recombinaison avec le site d'expression, soit parce que le gène ne peut coder pour un antigène fonctionnel. Une solution peut se présen-

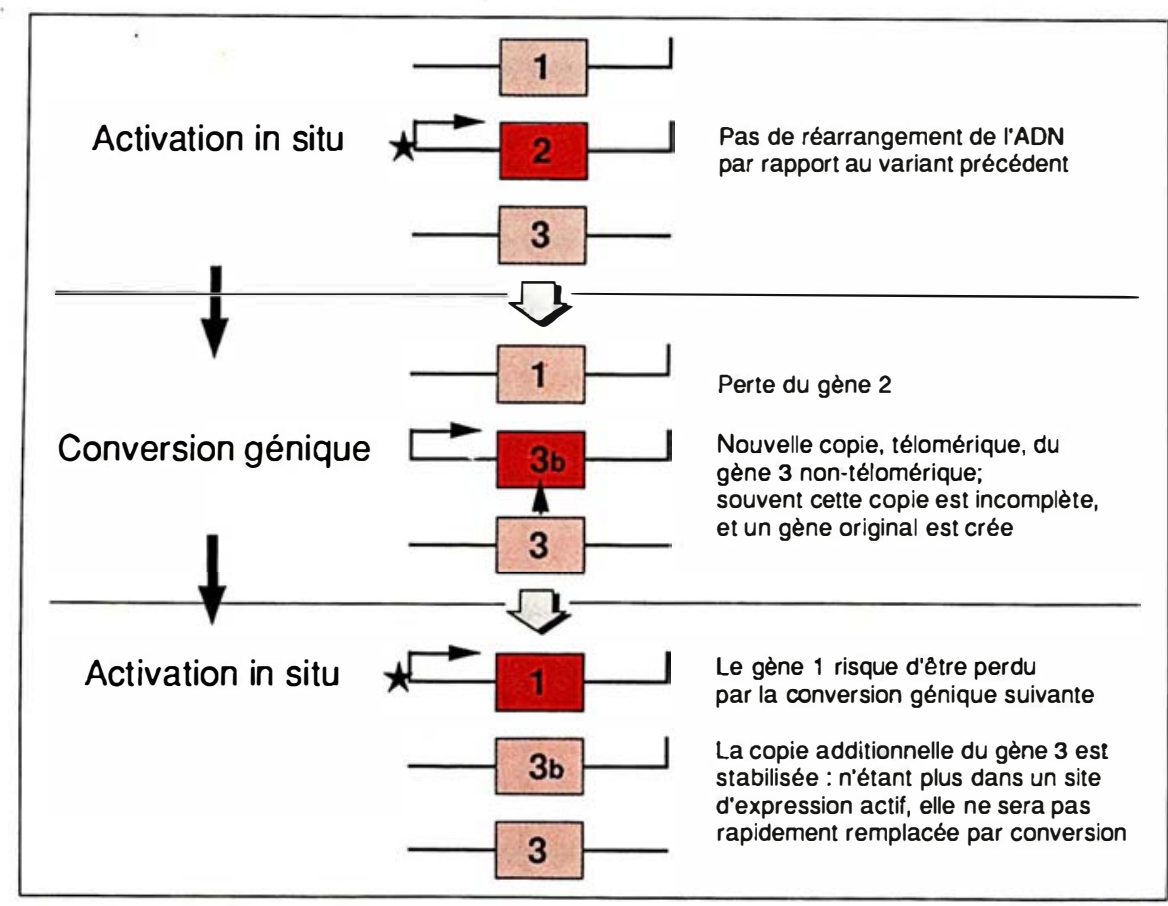

Figure 5. Évolution de familles de gènes de VSG par l'action des mécanismes de la variation antigénique. Trois gènes différents symbolisent le répertoire des gènes de VSG d'un clone, qui en réalité comprend plusieurs centaines d'unités. Les changements concernant ces trois gènes sont suivis au cours de trois événements de variation antigénique. L'activation du site $d^{\prime}$ expression est symbolisée par une étoile, et la conversion génique par une pointe de flèche. II faut remarquer que : 1) activation in situ et conversion génique n'alternent pas nécessairement; 2) seuls les événements recombinatoires productifs et donnant lieu à une variation antigénique peuvent être analysés; en réalité des recombinaisons "cryptiques " peuvent $s^{\prime} y$ ajouter et augmenter la dérive évolutive des gènes. 
ter lorsque le gène actif, présent dans le site d'expression, possède lui-même des homologies avec le donneur potentiel. Des recombinaisons dans ces zones d'homologie peuvent pallier les insuffisances, et engendrer des gènes fonctionnels. Ces événements recombinatoires sont nécessairement peu probables et tardifs, puisqu'ils dépendent de la nature du gène particulier présent dans le site d'expression. Par ailleurs, puisqu'ils se produisent au sein des gènes de VSG, ils conduisent à la formation de gènes composites. De tels gènes s'observent fréquemment tard dans l'infection chronique, et peuvent présenter une structure très complexe résultant de multiples événements recombinatoires, apparemment aléatoires. Il semble toutefois qu'en dehors des contraintes liées au changement des régions porteuses des épitopes antigéniques, des contraintes structurales favorisent les recombinaisons aboutissant à l'échange des domaines $\mathrm{N}$ - et C-terminaux.

\section{Évolution des répertoires antigéniques}

Les répertoires de VSGs sont étonnamment variables. Même entre isolats provenant d'une même région géographique, les types antigéniques communs (isotypes) sont minoritaires. Cette observation se reflète au niveau des gènes, à l'exception remarquable de certaines séquences non télomériques.

Curieusement, la sous-espèce T.b. gambiense, responsable de la maladie du sommeil en Afrique occidentale, échappe à cette règle. Dans ce cas particulier, les répertoires de gènes de VSG sont conservés entre stocks, même géographiquement très éloignés, ce qui en permet la discrimination aisée.

Entre autres facteurs possibles, l'évolution très rapide des répertoires antigéniques pourrait dépendre de l'utilisation alternée des différents mécanismes d'activation génique décrits ci-dessus (figure 5, page suivante). En effet, la succession " conversion génique - activation in situ" permet d'amplifier le nombre de copies d'un gène, puisque la copie additionnelle engendrée par conversion génique est du changement antigénique. Cette situation peut permettre la conservation, ne fût-ce que transitoire, de copies hybrides façonnées lors de recombinaisons complexes. En outre, cette copie, étant télomérique, peut faire partie du répertoire de gènes exprimés précocement, ce qui n'était pas le cas du gène originel si celuici était non télomérique. Inversement, la succession "activation in situ - conversion génique " conduit à la perte, par conversion, du gène présent à l'origine dans le site d'expression. Souvent de tels gènes télomériques sont présents en une seule copie dans le génome, et donc la perte du gène ne peut être compensée par l'expression d'un gène apparenté, ce qui conduit à la disparition irrémédiable du type antigénique correspondant. Ces différentes possibilités ont été vérifiées dans le laps de temps très réduit de l'expérimentation de laboratoire, qui ne reflète que de très loin la complexité de la situation sur le terrain. Dans le contexte de ces observations, la stabilité qui caractérise T.b. gambiense pourrait s'interpréter comme due à l'absence d'alternance dans l'utilisation des mécanismes d'activation génique, comme si cette sous-espèce ne disposait que de très peu de sites d'expression sanguicoles. Si cette interprétation est purement hypothétique, il semble acquis, en revanche, que la stabilité des répertoires d'antigènes chez T.b. gambiense s'étend aux types métacycliques. Cela ouvre certaines perspectives, bien lointaines il est vrai, dans l'optique d'une vaccination contre la maladie du sommeil.

\section{Conclusion}

L'analyse génétique du phénomène de la variation antigénique des trypanosomes avait été entreprise, à l'origine, afin d'en comprendre les mécanismes dans l'espoir de pouvoir les enrayer, et donc d'envisager des méthodes de lutte prophylactique contre les trypanosomiases. Si ces mécanismes sont maintenant mieux compris, il est clair que l'objectif initial est toujours hors d'atteinte. Le potentiel de variation du déterminant antigénique majeur, le VSG, semble déjouer l'attaque du chercheur aussi bien que la réponse du système immunitaire. En revanche, le trypanosome ne s'est pas montré avare d'informations, souvent remarquables. Si nous connaissons mieux comment sont structurés nos télomères, comment nos gènes d'antigènes varient ou comment ces antigènes s'ancrent dans la membrane plasmique, c'est en grande partie grâce à ce parasite exotique que nous le devons

\section{Summary}

Expression of variable antigen genes in African trypanosomes

African trypanosomes evade the immune response of their host by repeatedly changing their major surface antigen. Although several hundreds of different antigen genes exist in the genome, only one is usually expressed at a time. This expression occurs in one of several possible telomeric expression sites. Besides being exclusively telomeric, transcription of the antigen gene exhibits other particular characteristics: the RNA polymerase is unusually resistant to $\alpha$-amanitin, and the transcription unit comprises several other genes, one of which may encode an adenylate cyclase. Posttranscriptional controls modulate the activity of this transcription unit during the parasite life-cycle. Antigenic variation is achieved through either alternative activation of different expression sites, or gene recombination within a given, expression site. The succession of these mechanisms shapes the repertoires of antigen genes, and ensure a relative programming of antigen expression.

\section{TIRÉS A PART}

E. Pays. 